\title{
Fair Cellular Throughput Optimization with the Aid of Coordinated Drones
}

\author{
Edgar Arribas \\ IMDEA Networks Institute \\ Universidad Carlos III de Madrid \\ edgar.arribas@imdea.org
}

\author{
Vincenzo Mancuso \\ IMDEA Networks Institute \\ vincenzo.mancuso@imdea.org
}

\author{
Vicent Cholvi \\ Universitat Jaume I \\ vcholvi@uji.es
}

\begin{abstract}
The interest on flexible air-to-ground channels from aerial base stations to enhance users access by seeking good lineof-sight connectivity from the air has increased in the past years. In this paper, we propose a deployable analytical framework for the 3-D placement of a fleet of coordinated drone relay stations to optimize network capacity according to $\alpha$-fairness metrics. We formulate a mixed-integer non-convex program, which results to be intractable. Therefore, we design a near-optimal heuristic that can solve the problem in real-time applications. We assess the performance of our proposal by simulation, using a realistic urban topology, and study pros and cons of using drone relay stations in both static and dynamic scenarios, when popular events gather masses of users in limited areas.
\end{abstract}

\section{INTRODUCTION}

Cellular infrastructures are evolving towards flexible and reconfigurable solutions, able to cope with high densities of users. The fifth generation $(5 \mathrm{G})$ of networks [1] will include novel network paradigms, like smart and flexible relaying [2]. Precise beamforming and efficient cooperative transmission techniques will enable to operate broadband wireless backhaul links [3], which are key to promote the use of mobile relays.

Mobile relays can be mounted on vehicles and drones, so to move the network with the users, by moving relays where the fixed network cannot sustain the user demand [4]. Guo and O'Farrel [5] derived the capacity of OFDMA cellular networks like LTE/LTE-A in presence of relays reusing cellular frequencies. Although they do not model the use of drones, they show interference is the main limiting factor, so that relays like drones do need to be operated onto orthogonal frequencies, which gives additional advantages in terms of simplified resource allocation control [6]. Thus, we study the case of drones and base stations (BSs) transmitting on orthogonal bands.

Current works study optimal drone placement in oversimplified cases, neglecting inter-drone interference and fairness. We focus instead on the optimization of 3-D positions for a fleet of coordinated drone relays, as shown in Figure 1, based on the $\alpha$-fairness metric - a high-level generalization of fairness metrics to target maximum throughput, proportional fairness or max-min fairness in one framework [7]. We model random variations of signals, interference from relays and BSs, use of slotted time-frequency resources, cell selection and resource allocation schemes. Concretely, we adopt stochastic models for path-loss and availability of line-of-sight (LoS) and nonLoS (NLoS) links, and cast our problem into an OFDMA-like resource allocation scheme.

Finding the optimal drone positions is an NP-complete problem. However, we leverage a class of algorithms designed

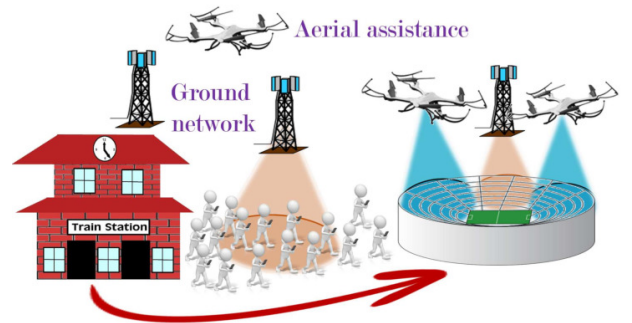

Figure 1: Reference scenario: multi-drone-aided network.

for optimization with intertwined variables: Extremal Optimization (EO) [8], which run in polynomial time. EO is based on iteratively picking the "least fit" element of a discrete set and change its parameters to improve the utility function.

We therefore formulate a suitable utility function, targeting $\alpha$-fair user throughput, and design PD-EOA: a PartiallyDistributed EO Algorithm that iteratively updates the position of the least fit drone, i.e., the drone relay that contributes the least to the utility function. Although EO is a form of centralized algorithms originally introduced for static optimization, we use such technique to design a partially distributed algorithm that works dynamically, as the system evolves.

We validate our proposal and assess its performance by simulations in realistic static and dynamic scenarios. As an illustration, we evaluate the performance of PD-EOA when users move towards a stadium before a sport event, so that their density grows over time. Our analysis unveils that the role of interference from drones and the stochastic characterization of LoS between drones and users make the optimization problem intractable, yet we are able to provide a near-optimal heuristic.

\section{RELATED WORK}

Non-terrestrial mobile relays have been investigated over several paradigms. Satellite networks [9] provide service to big areas, but users only achieve low rates upon high costs. Besides, satellites cannot adjust to users topology. In contrast, drone relays move dynamically at low altitudes and serve smaller target regions on demand, where the ground network cannot sustain the high demand from dense spots. The Loon project by Google [10] assesed the operation of aerial stations on high-altitude platforms (balloons), hovering some kilometers high and slowly drifting. Google provided basic network access to remote low-served areas. We instead focus on fleets of small relays flying at few hundreds of meters that serve broadband links and are easily repositioned on small time scales. Drone relays are also different from fixed relays and device-to-device approaches [11], since drone networks are 
neither fixed nor unplanned, and the channel propagation is impacted by the LoS state, which varies over time.

Thus, satellites, balloons or terrestrial relays cannot face scenarios as the ones we study, since protocol designs, technology constraints and signal propagation are radically different.

Moving drones to optimal locations to maximize various metrics is actively being investigated in the literature. AlHourani et al. [12] derived optimal altitude for one drone to maximize coverage, while Hayajneh et al. [13] derived optimum drone altitude to minimize outages and bit-error rate. Mozaffari et al. [14] found optimal locations for multiple noninterfering drones to minimize the total transmission power. Guo et al. [4] studied the generic use of drones as relays. Their approach provides more throughput in low-connectivity areas, but does not account for issues like spectral efficiency. Andryeyev et al. [15] estimate drone positions to increase cellular capacity through a self-organization algorithm based on repulsion from BSs and drones, and attraction by mobile users.

These works provide valuable and foundational contribution, but do not study problems like fair capacity maximization when drones assist cellular networks, neither provide realistic frameworks to integrate drone relays into current cellular networks, which is what we target with our approach.

\section{SYSTEM MODEL}

We derive an analytical framework to find optimal 3-D locations of drone relays, given the position of users and ground BSs. We target $\alpha$-fair instantaneous user rates by selecting drone positions as a function of the resource allocation policy adopted by ground BSs and drones the users can attach to.

\section{A. Reference Scenario}

We consider a ground surface $\mathcal{S}$ where a set $\mathcal{G}$ of $G$ ground BSs $(g B S)$ provide service to a set $\mathcal{U}$ of $U$ user equipments (UEs). The positions of each $g B S g$ and user $u$ are known and denoted as $\Pi^{g}=\left(X^{g}, Y^{g}\right)$ and $\pi_{u}=\left(x_{u}, y_{u}\right)$. We also consider a fleet $\mathcal{A}$ of $A$ aerial BSs $(a B S)$ as mobile relays, carried by drones. We assume the system operator offers user access through two bands, one used by $g B S$ s and another used by $a B S \mathrm{~s}$, so $a B S \mathrm{~s}$ and $g B S \mathrm{~s}$ do not interfere. We denote by $\Pi_{a}=\left(X_{a}, Y_{a}, h_{a}\right)$ the 3 -D position of each $a B S a \in \mathcal{A}$, and by $\mathcal{B}=\mathcal{G} \cup \mathcal{A}$ the set of all BSs $b \in \mathcal{B}$ that form the whole network.

We assume that $a B S$ s connect to the closest $g B S$ through wireless links with finite rate constraints. Operators usually install $g B S \mathrm{~s}$ on top of buildings or towers, so we assume that $g B S$ - $a B S$ links are free of obstacles. We denote the limit on the $g B S-a B S$ link capacity as $\tau_{a}$, and assume that it can be computed based on the position of $a \in \mathcal{A}$.

The interference from $a B S \mathrm{~s}$ affects the Signal-to-Noiseand-Interference Ratio (SINR) that UEs receive, and hence the air-to-ground link capacity changes depending on the $a B S$ positions. The propagation channel model from air to ground differs from conventional ground channels, and it is based on the LoS likelihood between $a B S$ s and users, as we detail next.

\section{B. Path-loss and Interference}

The conditions for the BS-UE access channels differ much depending whether users attach to a $g B S$ or an $a B S$. While the ground attenuation for $g B S$-UE links follows conventional path-loss models based on slow and fast fading, $a B S$-UE channels suffer additional attenuation depending on the LoS state, referred in the literature as an excess attenuation [16].

The LoS-likelihood is a complex function of the elevation angle between user $u \in \mathcal{U}$ and $a B S a \in \mathcal{A}$ :

$$
P_{L o S}(a, u)=\left(1+\beta_{1} \cdot e^{-\beta_{2}\left(\frac{180}{\pi} \arctan \left(\frac{h_{a}}{r_{a}, u}\right)-\beta_{1}\right)}\right)^{-1},
$$

where $h_{a}$ is the height of $a$, and $\beta_{1}$ and $\beta_{2}$ depend on the number of obstructions per unit area, buildings height, etc., as shown in [12], based on ITU recommendations [17]. In Eq. (1), $\arctan \left(h_{a} / r_{a, u}\right)$ is the elevation angle, derived from the $a B S$ height $h_{a}$ and the distance between user $u$ and the ground projection of drone $a, r_{a, u}=\left\|\left(X_{a}, Y_{a}\right)-\pi_{u}\right\|$. Safety, legislation and technology restrict drones to fly only at few hundreds of meters, so the ground distance has the same order as the drone height. Thus, the LoS varies sensibly with user and drone positions, and affect air-to-ground channel conditions.

The higher a drone hovers, the more likely it is to have LoS. However, the signal also attenuates with the distance and, in multi- $a B S$ scenarios as we discuss in this paper, interference from other $a B S \mathrm{~s}$ is an important parameter to consider. Thus, optimal drone hovering altitude depends on all $a B S \mathrm{~s}$.

As obtained in [12], the average attenuation (in $\mathrm{dB}$ units) of an air-to-ground channel is based on the LoS likelihood from Eq. (1). Hence, for all drone $a \in \mathcal{A}$ and user $u \in \mathcal{U}$ :

$$
\begin{aligned}
L_{\mathcal{A}}(a, u) & =20 \log _{10}\left(\frac{4 \pi f_{\mathcal{A}}}{c} \cdot \sqrt{h_{a}^{2}+r_{a, u}^{2}}\right)+ \\
& +P_{L o S}(a, u) \cdot\left(\xi_{L o S}-\xi_{N L o S}\right)+\xi_{N L o S},
\end{aligned}
$$

where $\xi_{L o S}, \xi_{N L o S}$ are the excess attenuation components discussed above in LoS and NLoS conditions respectively, $f_{\mathcal{A}}$ is the carrier band in $\mathrm{Hz}$ and $c$ is the speed of light in $\mathrm{m} / \mathrm{s}$.

Ground $g B S$-UE links experience an attenuation based on the well known path-loss model with slow fading (in $\mathrm{dB}$ units):

$$
L_{\mathcal{G}}(g, u)=10 \eta_{\mathcal{G}} \log _{10}\left(\frac{4 \pi f_{\mathcal{G}}}{c} \cdot\left\|\Pi^{g}-\pi_{u}\right\|\right)+\mathcal{N}\left(0, \sigma_{\mathcal{G}}^{2}\right),
$$

where $\eta_{\mathcal{G}}>2$ is the ground path-loss exponent, $f_{\mathcal{G}}$ is the carrier band in $\mathrm{Hz}$, and $\sigma_{\mathcal{G}}$ is the standard deviation of the gaussian variable $\mathcal{N}\left(0, \sigma_{\mathcal{G}}^{2}\right)$, modelling the effects of shadowing.

To measure the network capacity, we consider that the BSs serve ground users with the rate corresponding to the Shannon capacity, according to the SINR of the link. The bands used by $g B S \mathrm{~s}$ and $a B S \mathrm{~s}$ have bandwidth of $W_{\mathcal{G}}$ and $W_{\mathcal{A}}$, respectively.

With the above, SINRs for links $(g, u)$ and $(a, u)$ are:

$\gamma_{g, u}^{\mathcal{G}}=\frac{P_{T x}^{g} \cdot 10^{-L_{\mathcal{G}}(g, u) / 10}}{N_{g, u}+I_{g, u}^{\mathcal{G}}}, \quad \gamma_{a, u}^{\mathcal{A}}=\frac{P_{T x}^{a} \cdot 10^{-L_{\mathcal{A}}(a, u) / 10}}{N_{a, u}+I_{a, u}^{\mathcal{A}}}$,

where $P_{T x}^{g}$ and $P_{T x}^{a}$ are the transmission power of the BSs $g \in \mathcal{G}$ and $a \in \mathcal{A}$, respectively, $N_{g, u}$ and $N_{a, u}$ represent thermal noise, and $I_{g, u}^{\mathcal{G}}$ and $I_{a, u}^{\mathcal{A}}$ are interferences that user $u$ suffers from $g B S \mathrm{~s}$ or $a B S \mathrm{~s}$, respectively. While for ground connections the position of the interfering $g B S$ in $\mathcal{G} \backslash\{g\}$ is fixed and known so that the interference $I_{g, u}^{\mathcal{G}}$ is a constant value, ${ }^{1}$ the $3-\mathrm{D}$ position of an $a B S$ is variable and directly affects the interfering signals received by user $u$, i.e.:

$$
I_{a, u}^{\mathcal{A}}=\sum_{a^{\prime} \in \mathcal{A} \backslash\{a\}} P_{T x}^{a} \cdot 10^{-L_{\mathcal{A}}\left(a^{\prime}, u\right) / 10}, \quad \forall a \in \mathcal{A} .
$$

${ }^{1}$ Average interference in $g B S$ s is fixed because $g B S$ s do not move, although instantaneous values are random fluctuations from slow and fast fading. 


\section{Cell Selection and Resource Allocation}

BSs cannot provide service to unlimited users. Hence, we assume that each $g B S$ or $a B S$ can serve $U_{\max }$ users at most. Users perform cell selection as in LTE networks, before the optimization: first, UEs select the BS with strongest Signal-toNoise Ratio (SNR); if the link request is rejected, then the UE performs cell re-selection, and tries to attach to the BS with next strongest SNR observed, until the user gets attached. We denote the $g B S$ to which user $u$ attaches as $g_{u}$. This best-SNR policy is the one currently adopted in LTE networks [18].

We assume that $g B S \mathrm{~s}$ and $a B S \mathrm{~s}$ schedule users using an OFDMA system (like in LTE). BSs have a finite set of physical resource blocks (PRB) - the smallest unit of time-frequency resources-organized in subframes. Thus, the minimum bandwidth allocated to a user is the bandwidth of one PRB per frame. We denote as $W_{\mathcal{G}}^{\mathrm{min}}$ and $W_{\mathcal{A}}^{\mathrm{min}}$ the minimum bandwidth that a $g B S$ or an $a B S$ can allocate to a single user.

\section{OPTIMAL FAIR THROUGHPUT BY DRONE POSITIONING}

Here we derive an analytic framework for the 3-D positions of $a B S \mathrm{~s}$, to optimize throughputs based on $\alpha$-fairness. Depending on the value of $\alpha \geq 0$ - the fairness level-we capture different fairness criterias such as proportional fairness $(\alpha=1)$, max-min fairness $(\alpha \rightarrow+\infty)$ or the maximum capacity $(\alpha=0)$.

Denoting by $T_{b, u}$ the access link throughput between BS $b \in \mathcal{B}=\mathcal{G} \cup \mathcal{A}$ and user $u \in \mathcal{U}$, we have the following utility:

$$
U_{t h r}^{\alpha}= \begin{cases}\frac{1}{1-\alpha} \cdot \sum_{u \in \mathcal{U}}\left(\sum_{b \in \mathcal{B}} T_{b, u}\right)^{1-\alpha}, & \alpha \neq 1 ; \\ \sum_{u \in \mathcal{U}} \log \left(\sum_{b \in \mathcal{B}} T_{b, u}\right), & \alpha=1 .\end{cases}
$$

Throughput Problem $\mathcal{T}$ : Given $G$ gBSs, A aBSs hovering at heights in the range $\left[h_{\min }, h_{\max }\right], U$ UEs that may connect to either a $g B S$ or an aBSs, each of which serves $U_{\max } U E s$ at most, and a backhaul capacity $\tau_{a}$, find the optimal position of aBSs, optimal user association, and the optimal user resource allocation so to maximize the $\alpha$-fair throughput utility.

We denote as $C_{a, u}$ and $C_{u}^{g_{u}}$ the binary decision variables that tell whether $u \in \mathcal{U}$ connects to $a \in \mathcal{A}$ or $g B S g_{u}$. Variables $T_{b, u}$ and $W_{b, u}$ denote the throughput and bandwidth allocated to access link $(b, u)$. The resulting optimization program is:

$$
\begin{cases}\max _{C_{a}, u}, C_{u}^{g u}, \Pi_{a}, T_{b, u}, W_{b, u} \quad U_{t h r}^{\alpha} & \forall u \in \mathcal{U} ; \\ C_{u}^{g_{u}}+\sum_{a \in \mathcal{A}} C_{a, u}=1, & \forall u \in \mathcal{U} ; \\ T_{g_{u}, u}=W_{g_{u}, u} \cdot \log _{2}\left(1+\gamma_{g_{u}, u}^{\mathcal{G}}\right), & \forall u \in \mathcal{U} ; \\ W_{\mathcal{G}}^{\min } \cdot C_{u}^{g_{u}} \leq W_{g_{u}, u} \leq W_{\mathcal{G}} \cdot C_{u}^{g_{u}} & \forall g \in \mathcal{G} ; \\ \sum_{u \in \mathcal{U}} W_{g, u} \leq W_{\mathcal{G}} ; & \forall a \in \mathcal{A}, \forall u \in \mathcal{U} ; \\ T_{a, u}=W_{a, u} \cdot \log _{2}\left(1+\gamma_{a, u}^{\mathcal{A}}\right), & \forall a \in \mathcal{A}, \forall u \in \mathcal{U} ; \\ W_{\mathcal{A}}^{\min } \cdot C_{a, u} \leq W_{a, u} \leq W_{\mathcal{A}} \cdot C_{a, u}, & \forall a \in \mathcal{A} ; \\ \sum_{u \in \mathcal{U}} W_{a, u} \leq W_{\mathcal{A}}, & \forall g \in \mathcal{G}, \forall a \in \mathcal{A} ; \\ \sum_{u \in \mathcal{U}} T_{a, u} \leq \tau_{a}, \quad C_{u}^{g} \leq U_{\max }, \sum_{u \in \mathcal{U}} C_{a, u} \leq U_{\max }, & \forall a \in \mathcal{A} .\end{cases}
$$

The first constraint states that each $u \in \mathcal{U}$ connects only to one BS. The second constraint gets the throughput allocated to $g B S$ s. The third and fourth constraints get the bandwidth allocated to each user of $g B S \mathrm{~s}$, with minimum guarantees. The fifth constraint gets the air-to-ground link rates, and the sixth and seventh constraints get the bandwidth allocated by $a B S \mathrm{~s}$, with minimum guarantees. The eighth and ninth constraints limit the aggregate transmission rate of $a B S \mathrm{~s}$ to their backhaul capacity, and the maximum allowed served users by each BS, respectively. The last constraint delimits the 3-D space within a ball $\mathcal{S}_{a}$ centered on the current position of the drone.

The formulated problem is mixed-integer and non-convex, hence not tractable with currently available optimizers. Note in fact that air-to-ground links bring unavoidable non-convex functions. Finding the optimal drone positions is an NPComplete problem. Indeed, the NP-Complete MinimumGeometric Disk-Cover (MGDC) problem [19] can be reduced, in polynomial time, to a special instance of the problem where users get $1 \mathrm{bps}$ if a drone serves them and 0 bps otherwise. We omit the details due to lack of space.

\section{PARtially-Distributed EXtremal Optimization}

Problem $\mathcal{T}$ is hard to solve since any change in a decision variable affects association and resource allocation of all users. This is the kind of problems EO has been thought for. Hence, in order to find time-efficient and near-optimal solutions, we propose PD-EOA, a partially-distributed EO algorithm. PDEOA is based on the three decisions that the framework makes: ( $i$ ) 3-D $a B S$ positions; ( $i i)$ sets of UEs attached to each $g B S$ and $a B S$; and (iii) bandwidth allocation from each $g B S$ or $a B S$ to their attached UEs. PD-EOA iteratively solves these three steps: step $(i)$ is solved centrally with EO, step $(i i)$ is solved independently by users, and step ( $i i i)$ is optimally solved distributedly by each $g B S$ and $a B S$, as detailed next.

The pseudocode of PD-EOA is shown in Algorithm 1. The algorithm starts from an initial random feasible implementation of the system. Let $\mathcal{U}_{g}$ and $\mathcal{U}_{a}$ be the sets of users attached to $g B S g$ and $a B S a$, respectively. We can split the utility into a utility provided by $g B S \mathrm{~s}\left(U_{\mathcal{G}}^{\alpha}\left(\mathcal{U}_{g}\right)\right)$ and by $a B S \mathrm{~s}\left(U_{\mathcal{A}}^{\alpha}\left(\mathcal{U}_{a}\right)\right)$. The $a B S$ that provides the worst utility is $a_{w}=\arg \min _{a \in \mathcal{A}} U_{\mathcal{A}}^{\alpha}\left(\mathcal{U}_{a}\right)$, referred as the "least fit" $a B S$.

At each iteration, PD-EOA identifies and moves the least fit $a B S$ to a position where the system utility increases the most ( $\operatorname{step}(i)$ ). To this end, the algorithm explores candidate positions within a ball $\mathcal{S}_{a}$ and only considers the points of a 3-D lattice that spans equal-volume subspaces.

User attachment (step $(i i)$ ) is based on SNR, so it is performed by users and BSs distributedly: each user requests connection to the BS that offers the best SNR. If accepted, it is associated to the $\mathrm{BS}$, otherwise it requests association to the BS with the next-in-list best SNR.

Finally, since BS utilities are additive, each $g B S$ and $a B S$ can address the bandwidth allocation optimally on its own (step $($ iii $)$ ). Specifically, given a BS $b \in \mathcal{B}=\mathcal{G} \cup \mathcal{A}$ and its position, the set of attached users $\mathcal{U}_{b}$, and the spectral efficiency of users computed with the Shannon formula, we obtain the following Convex Program (CP) for resource allocation:

$$
\left\{\begin{array}{l}
\max _{w_{u}} \frac{1}{1-\alpha} \cdot \sum_{u \in \mathcal{U}_{b}}\left(W_{\mathcal{V}} \cdot w_{u} \cdot \log _{2}\left(1+\gamma_{b, u}^{\mathcal{V}}\right)\right)^{1-\alpha} \\
\sum_{u \in \mathcal{U}_{b}} w_{u}=1 ; \\
w_{u} \geq W_{\mathcal{V}}^{\min } / W_{\mathcal{V}}, \quad \forall u \in \mathcal{U}_{b} ;
\end{array}\right.
$$

where $\mathcal{V} \in\{\mathcal{G}, \mathcal{A}\}$, and $w_{u}$ is the fraction of the total bandwidth that BS $b$ allocates to $u$. The first constraint ensures that all the bandwidth is used. The second constraint ensures that each user obtains at least the minimum bandwidth guaranteed. 
TABLE I: OPTIMAL SOLUTION FOR THE CONVEX PROGRAM (8)

\begin{tabular}{|c|c|c|c|}
\hline \multicolumn{4}{|l|}{$\alpha=0$} \\
\hline$w_{u}=$ & $\begin{array}{l}W_{\mathcal{V}}^{\min } / W_{\mathcal{V}}, \\
1-\left(\left|\mathcal{U}_{b}\right|-1\right) \cdot W_{\mathcal{V}}^{\min } / W_{\mathcal{V}}\end{array}$ & \multicolumn{2}{|c|}{$\begin{array}{l}u \neq \arg \max _{v \in \mathcal{U}_{b}} \theta_{v} ; \\
u=\arg \max _{v \in \mathcal{U}_{b}} \theta_{v} ;\end{array}$} \\
\hline$\alpha \in] 0,1[;$ & \multirow{2}{*}{$\frac{\theta_{u}^{1 / \alpha-1}}{\sum_{v \in \mathcal{U}_{b}} \theta_{v}}, \quad \forall u \in \mathcal{U}_{b}$} & $\alpha=1$ & \\
\hline$w_{u}=$ & & $w_{u}=\frac{1}{\left|\mathcal{U}_{b}\right|}$, & $\forall u \in \mathcal{U}_{t}$ \\
\hline$\alpha \rightarrow+\infty$ & \multirow{2}{*}{$\begin{array}{l}W_{\mathcal{V}}^{\min } / W_{\mathcal{V}} \\
\frac{1-\left(\left|\mathcal{U}_{b}\right|-|\mathcal{J}|\right) \cdot W_{\mathcal{V}}^{\min } / W_{\mathcal{V}}}{\theta_{\mathcal{u}} \cdot \sum}\end{array}$} & & \\
\hline$w_{u}$ & & $\begin{array}{l}\text { if } u \notin \mathcal{J} \\
\text { if } u \in \mathcal{J}\end{array}$ & \\
\hline
\end{tabular}

\begin{tabular}{l}
\hline Algorithm 1 PD-EOA \\
\hline Require: Lattice $\mathcal{L}$, fleet $\mathcal{A}$, users $\mathcal{U}$, and Signal parameters. \\
1: Randomly place all $a$ at $\Pi^{a} \in \mathcal{L}$. Define $\Pi=\left\{\Pi^{a}\right\}_{a \in \mathcal{A}}$. \\
2: Obtain set $\mathcal{U}_{b}(\Pi)$ of users that connect to each $b \in \mathcal{B}$. \\
3: $U_{\mathcal{B}}^{\alpha}\left(\mathcal{U}_{b}(\Pi)\right) \leftarrow \operatorname{optimal} \alpha$-utility of each $b \in \mathcal{B}$ from $\mathrm{CP}(8)$. \\
4: Select $a_{0}=\arg \min _{a \in \mathcal{A}}\left\{U_{\mathcal{B}}^{\alpha}\left(\mathcal{U}_{b}(\Pi)\right)\right\}$. \\
5: Take $\Pi^{a_{0}}=\arg \max _{\pi \in \mathcal{L}}\left\{U_{t h r}^{\alpha}\right\}$ and place $a_{0}$ at $\Pi^{a_{0}}$. \\
6: Go back to step 2 until: \\
• No better position is found for $a B S a_{0}$. \\
- Maximum number of iterations $\kappa$ is reached. \\
\hline
\end{tabular}

CP (8) is convex when $\alpha \in[0,1[, \alpha \rightarrow 1$ or $\alpha \rightarrow+\infty$, which are the cases of interest of this paper. Thus, we derive KKT conditions [20] and find a closed-form solution. The KKT approach is well-known and extensively studied. Thus, we directly show the solution in Table I, where computing $\mathcal{J}$ when $\alpha \rightarrow+\infty$ takes linear time with respect to $\left|\mathcal{U}_{b}\right|$, and is equal to $\mathcal{J}=\left\{u \in \mathcal{U}_{b} \mid w_{u} \cdot \theta_{u}=\min _{v \in \mathcal{U}_{b}} w_{v} \cdot \theta_{v}\right\}$.

$\mathrm{PD}-\mathrm{EOA}$ is fast and requires few iterations. We have performed all the optimizations reported in less than 1 second on a personal computer. Thus, PD-EOA suits for on-demand optimization to dynamically reposition drones to adapt to user's movements over time. The complexity is $\mathcal{O}\left(|\mathcal{L}| \cdot U_{\max }\right)$.

\section{Numerical Simulations}

Here we show numerical simulations of the proposed framework in static and dynamic cases over the real topology of a dense city covered by $10 \mathrm{gBS}$, serving 1000 users. We validate $\mathrm{PD}-\mathrm{EOA}$ in comparison with optimal results approximated with Monte-Carlo (MC) simulations in small networks and study three significative static and dynamic scenarios:

- PPP: We place UEs through a Poisson point process.

- Stadium: we statically place $60 \%$ of the UEs in the surroundings of a stadium, and the rest like in PPP.

- Event: $40 \%$ of the UEs move according to the random way-point model. Rest of UEs arrive at an official scheduled rate of a train station and move towards the stadium.

We study drones placement, throughput and system fairness and show results for three cases: $(i)$ for $\alpha=0$, we get the maximum throughput (MaxThr), (ii) for $\alpha=1$, we optimize proportional fairness (PropFair), and (iii) for $\alpha \rightarrow+\infty$, we optimize the max-min fairness (MaxMin). We use these $\alpha$-values also for the ground network in absence of drones (Ground) and to compare PD-EOA to a state-ofthe-art proposal: the Repulsion-Attraction scheme (RA) [15]. Error bars in the figures are $95 \%$ confidence intervals. Table II gathers the used parameters. We use MATLAB R2018a to simulate channel conditions and optimize $a B S$ positions.
TABLE II: EVALUATION PARAMETERS

\begin{tabular}{|c|c|}
\hline Parameter & Value \\
\hline \hline$\xi_{L o S}, \xi_{N L o S}, \beta_{1}, \beta_{2}$ & $1.6 \mathrm{~dB}, 23 \mathrm{~dB}, 12.08,0.11$ \\
\hline Carrier frequencies, $f_{\mathcal{G}}, f_{\mathcal{A}}$ & $1815.1 \mathrm{MHz}, 2.63 \mathrm{GHz}$ \\
\hline Bandwidths, $W_{\mathcal{G}}, W_{\mathcal{A}}$ & $18 \mathrm{MHz}, 18 \mathrm{MHz}$ \\
\hline Tx power, $P_{T x}^{g}, P_{T x}^{a}$ & $44 \mathrm{dBm}, 25 \mathrm{dBm}$ \\
\hline Ground path loss exponent, $\eta_{\mathcal{G}}$ & 3 \\
\hline Height range, $\left[h_{\min }, h_{\max }\right]$ & {$[40,300] \mathrm{m}$} \\
\hline Urban area, $|\mathcal{S}|$ & $10 \mathrm{~km}$ \\
\hline Average walking speed & $2.5 \mathrm{~m} / \mathrm{s}$ \\
\hline Monte-Carlo runs per instance & $10^{7}$ \\
\hline Instances of simulations & 1000 \\
\hline
\end{tabular}

\section{A. Validation of PD-EOA operation}

We compare PD-EOA and optima in the PPP case. Figure 2 shows that utilities from PD-EOA and MC only differ by $1 \%$. Similarly, Figure 3 shows that throughput is like in the optimal case. Fairness, not shown here for lack of space, is nearoptimal as well. The figures also unveil that utility grows linearly with the fleet size, and the gain can be significantly large.

Utility and throughput increase with the fleet size because more capacity, connectivity and placement options appear, despite the interference. MaxThr is always unfair (Jain's index is below 0.05) because it allocates one resource block to each user and the rest of resources to users with best channels. Coversly, PropFair and MaxMin provide less throughput, but compensate with higher fairness, above 0.65 and 0.95 (not shown due to lack of space). Placing drones with PropFair or MaxMin does not hurt fairness, yet only a limited number of users benefit from them, which may generate undesired behaviors. The gain with respect to Ground is remarkable, and confirms that drone relays have huge potentials.

\section{B. Performance evaluation in the static Stadium case}

The Stadium case described here studies the network when people gather in dense spots. Here, $60 \%$ of users are in a stadium: $600 \mathrm{UEs}$, which is a typical case of users allocated over the same LTE band by the same operator (i.e., we take a stadium of 12000 seats, 4 operators, 3 LTE bands, and $60 \%$ of the attendance connected).

Results here show that increasing the fleet size is not always beneficial. In Figure 4, utility quickly saturates for MaxMin. Figure 5 reveals the reasons of such behavior by zooming into the performance of stadium users and rest of users. By adding drones, MaxThr and MaxMin do not necessarily improve throughput for stadium users, and even incur losses. Globally, with MaxThr it is still convenient to add drones, as they find some user with poor signal and so inject him high throughput. With MaxMin, clustering drones by the stadium is not convenient as well, because of interference that induces low quality at some user. PD-EOA with PropFair behaves differently because its mathematical design does not let fairness decay. Having many users in a dense spot is a key chance for $a B S \mathrm{~s}$ to increase capacity and fairness. Hence, PropFair brings drones by the stadium, so the utility increases with the number of drones while the fairness values remain at high values. We also see that PropFair outperforms all schemes in terms of throughput of users by the stadium. In fact, a few drones are not enough to boost throughput with MaxMin, and increasing the number of drones hovering the stadium adds interference, which is why we see no gain in the performance of MaxMin. 


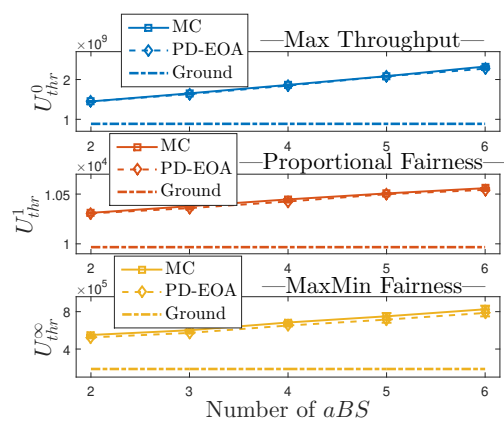

Figure 2: Utility validation for $\alpha \in\{0,1, \infty\}$. $G=10, U=750$. Scenario: PPP.

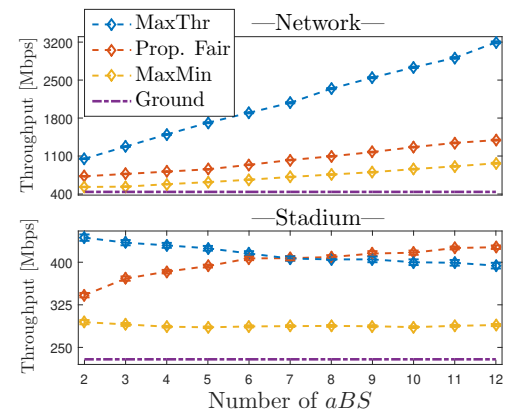

Figure 5: Network (upper) and Stadium (lower) throughput for $\alpha \in\{0,1, \infty\}$. $U=1000$ Scenario: Stadium with $U_{d}=600$.

This evaluation tells that $a B S \mathrm{~s}$ are beneficial to help the ground network in dense spots, except they cannot purely maximize throughput. They cannot either "rescue" all users with bad channel conditions, but can always provide fairness.

\section{Performance evaluation in a dynamic event case}

In this scenario, masses of 40 users arrive to the train station every 5 minutes. The initial population is 400 users and grows during 75 minutes up to 1000 users. There are $5 \mathrm{aBSs}$ hovering the area. Upon a train arrival, users walk towards the stadium, located $1.5 \mathrm{~km}$ away. We use PropFair to compute drone positions, since it conveys a good tradeoff of throughput and fairness. Drones reposition every 5 minutes with PD-EOA.

As time passes by, e.g., after $30 \mathrm{~min}$. of train arrivals, some people have reached the stadium, while many others keep walking towards it. At this point, the fleet is ready to serve the users by the stadium and also the smaller masses of people that are by the train station or on their way to the event. After one hour, drones have repositioned, as shown in Figure 6, where the mass at the stadium is much denser and more drones come to assist the users by the stadium. The trajectories of drones over 75 minutes are shown in Figure 7: the network tracks users and dispatches drones accordingly. $a B S 1$ is not required to assist the dense spot; $a B S \mathrm{~s} 2$ and 3 keep moving back and forth between the train station and the stadium; and $a B S \mathrm{~s} 4$ and 5 move within different regions, to fairly supply the users.

Figures 8-10 illustrate performance over time. We compare PD-EOA to the RA scheme proposed in [15], in which $a B S \mathrm{~s}$ are attracted by UE's inverse SNR, and repulsed by proximity to gBSs to avoid interference. The figures only consider performance of users arriving over time (i.e., the attendance).

As the attendance increases, we observe in Figure 8 a utility raise with MaxThr and PropFair, since both are opportunistic schemes. With MaxThr, the gain of PD-EOA over RA and Ground is high, although it saturates quickly. Instead,

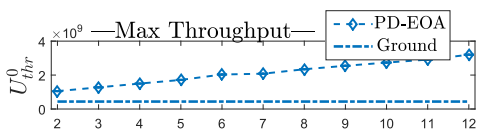

$1.4 \times 10^{4}-$ Proportional Fairness-

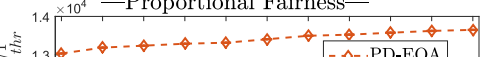

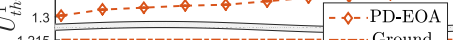

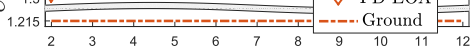
$\times 10^{4} \quad$-MaxMin Fairness-

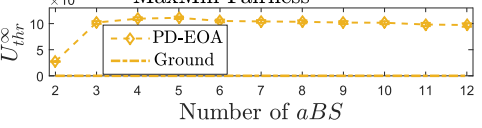

Figure 4: Network utility for $\alpha \in\{0,1, \infty\}$. $U=1000$. Scenario: Stadium with $U_{d}=600$.

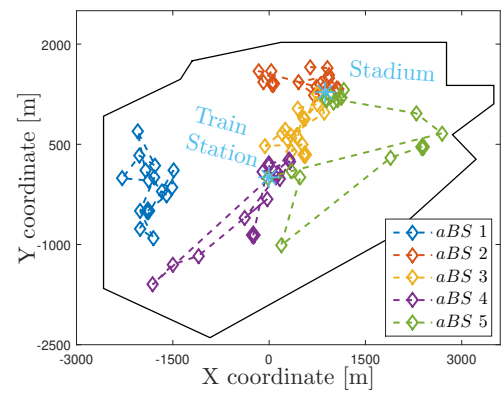

Figure 7: Drone paths for $75 \mathrm{~min} . A=5, U=$ [400, . . , 1000]. Scenario: PropFair, Event.

with PropFair, PD-EOA exhibits a smoother behavior, as its gain keeps increasing. In contrast, with MaxMin we observe a decay of performance over time, since the higher the number of users, the lower the minimum achieved rate.

In all cases, the aggregated throughput increases for all schemes and $\alpha$-values, as shown in Figure 9. PropFair exhibits a similar-slightly better in most of the cases-increase than MaxThr. However, the attendance grows linearly over time, while the throughput does not. This occurs because a fixed number of drones cannot serve unlimited users. Clearly, the RA scheme is not able to opportunistically take advantage of user's diversity and improve utility because it does not target a throughput-based metric. In contrast, PropFair with PDEOA provides better throughput and fairness guarantees.

Finally, Figure 10 shows the fairness achieved over time by the attendance. PD-EOA with PropFair and MaxMin provide better fairness, with values over 0.63 in all cases. In particular, with a proportionally-fair resource allocation, PDEOA can provide up to a $20 \%$ more fairness than RA.

\section{LESSONS LEARNT AND DISCUSSION}

Our results show the importance of integrating a fleet of drone relay stations in a cellular network. They also unveil that optimizing drone positions to boost throughput, without considering fairness, has little relevance in presence of dense spots of ground users. In contrast, a fair metric like PropFair provides relevant throughput improvement opportunities and achieves fair behaviors. Other metrics like MaxMin are useful for cases not addressed in this paper, e.g., to bring minimal communications in case of temporary loss of infrastructure.

The key of our scheme is the combination of centralized drone positions and distributed resource allocation, whereas users freely attach to their preferred BS according to a known policy (best signal, in this paper). With that, our partiallydistributed scheme, PD-EOA, uses a few iterations to compute 

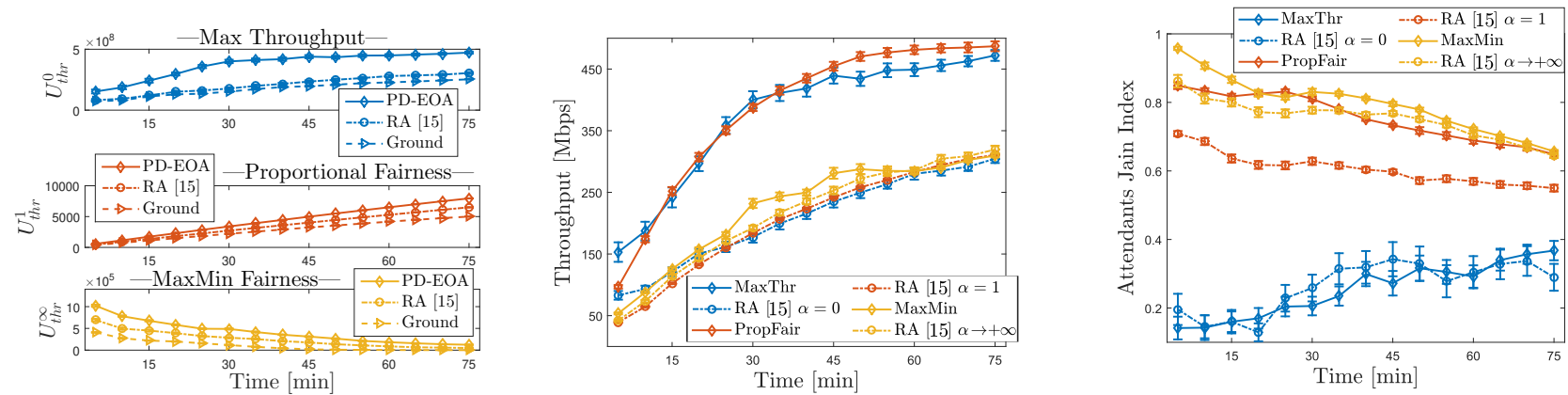

Figure 8: Attendance utility for $\alpha \in\{0,1, \infty\}$. Figure 9: Attendance capacity for $\alpha \in\{0,1, \infty\}$. Figure 10: Attendance fairness for $\alpha \in\{0,1, \infty\}$. $A=5, U=[400, \ldots, 1000]$. Scenario: Event. $A=5, U=[400, \ldots, 1000]$. Scenario: Event.

$A=5, U=[400, \ldots, 1000]$. Scenario: Event.

optimal positions for a fixed $\alpha$, which states the fairness degree in the network. Since PD-EOA is fast, it is possible to design an almost continuous repositioning in realistic networks.

The actual implementation of PD-EOA needs further work. It is clear that it requires exchanging signaling information between drones and a centralized orchestrator, and implementing a mechanism to track users. Signaling (out of the scope of this paper) incurs some overhead, depending on the frequency of reconfigurations, to gather positions and instruct drones.

Another constraint that might hinder the performance of drone relays is the backhaul capacity, i.e., the bandwidth that drones can use to accommodate the demand of users towards the core of the network. There are promising technologies to avoid bottlenecks on such backhaul, e.g., based on mmWave in 5G [21]. This is left for future work, yet our study already analytically accounts for backhaul bandwidth constraints.

Regarding drone mechanicals, commercial drones can carry small BSs, although they can fly for short time due to battery limitations ( $\sim 30 \mathrm{~min}$.). They can move at speeds of $15 \mathrm{~m} / \mathrm{s}$, so it is possible to derive a repositioning scheme that replaces drones that recharge on dedicated charging stations. For the case of Section VI-C, the routes flown by drones are short enough, and they can hover a city district in few minutes. Thus, notwithstanding the intricacies of the analysis, the performance evaluation discussed is relevant for realistic systems.

\section{CONCLUSIONS}

In this paper we have proposed an analytic framework to optimize drone-aided cellular networks in terms of an $\alpha$-fair throughput utility function under realistic stochastic models. Specifically, we have studied the integration of a coordinated fleet of aerial base stations to complement the service offered by ground base stations. Due to the complexity of the studied problem, we resorted to a heuristic, and so designed PD-EOA, a partially distributed algorithm based on extremal optimization. PD-EOA performs near-optimally in polynomial time, which makes it suitable for implementation in dynamically changing environments. The performance evaluation presented shows that PD-EOA brings significant gain and outperforms existing approaches. It also unveils that using fairness is key to get benefit from coordinated yet interfering drone relays.

\section{ACKNOWLEDGMENTS}

The work of E. Arribas is partially supported by the FPU15/02051 grant from the Spanish Ministry of Education, Culture and Sports (MECD). The work of V. Mancuso is supported by the Ramón y Cajal grant (ref: RYC-2014-16285) from the Spanish Ministry of Economy and Competitiveness and by the DiscoEdge grant (TIN2017-88749-R) from the Spanish Ministry of Science, Innovation and Universities.

\section{REFERENCES}

[1] W. Mohr, "5G empowering vertical industries," Tech. Rep., April 2016.

[2] M. Maternia et al., "5G PPP use cases and performance evaluation models," 5G-PPP, Tech. Rep., Apr. 2016, White Paper.

[3] C. Liu, K. Sundaresan, M. Jiang, S. Rangarajan, and G. Chang, "The case for re-configurable backhaul in cloud-RAN based small cell networks," in IEEE INFOCOM, 2013.

[4] W. Guo, C. Devine, and S. Wang, "Performance analysis of micro unmanned airborne communication relays for cellular networks," in IEEE CSNDSP. IEEE, 2014.

[5] W. Guo and T. O'Farrell, "Relay deployment in cellular networks: Planning and optimization," IEEE JSAC, vol. 31, no. 8, 2013.

[6] M. Mozaffari, W. Saad, M. Bennis, and M. Debbah, "Wireless communication using unmanned aerial vehicles (UAVs): Optimal transport theory for hover time optimization," IEEE Transactions on Wireless Communications, vol. 16, no. 12, pp. 8052-8066, 2017.

[7] L. Buzna and R. Carvalho, "Controlling congestion on complex networks: fairness, efficiency and network structure," Scientific reports, vol. 7, no. 1, p. 9152, 2017.

[8] S. Boettcher and A. Percus, "Optimization with extremal dynamics," Phys. Rev. Lett., vol. 86, pp. 5211-5214, 2001.

[9] R. Ferrús, H. Koumaras, O. Sallent, G. Agapiou, T. Rasheed, M.-A Kourtis, C. Boustie, P. Gélard, and T. Ahmed, "SDN/NFV-enabled satellite communications networks: Opportunities, scenarios and challenges," Physical Communication, vol. 18, pp. 95-112, 2016.

[10] S. Katikala, "Google ${ }^{\mathrm{TM}}$ project loon," InSight: Rivier Academic Journal, vol. 10, no. 2, pp. 1-6, 2014

[11] E. Arribas and V. Mancuso, "Multi-path D2D leads to satisfaction," in IEEE 18th International Symposium on A World of Wireless, Mobile and Multimedia Networks (WoWMoM). IEEE, 2017, pp. 1-7.

[12] A. Al-Hourani, S. Kandeepan, and S. Lardner, "Optimal LAP altitude for maximum coverage," IEEE Wireless Communications Letters, vol. 3 , no. 6 , pp. 569-572, 2014

[13] A. Hayajneh, S. Zaidi, D. McLernon, and M. Ghogho, "Optimal dimensioning and performance analysis of drone-based wireless communications," in GC Wkshps. IEEE, 2016.

[14] M. Mozaffari, W. Saad, M. Bennis, and M. Debbah, "Optimal transport theory for power-efficient deployment of unmanned aerial vehicles," in IEEE ICC 2016, 2016.

[15] O. Andryeyev and A. Mitschele-Thiel, "Increasing the cellular network capacity using self-organized aerial base stations," in DroNet. ACM, 2017, pp. 37-42.

[16] A. Al-Hourani, S. Kandeepan, and A. Jamalipour, "Modeling air-toground path loss for low altitude platforms in urban environments," in GLOBECOM. IEEE, 2014, pp. 2898-2904.

[17] ITU-R Recommendations, "Propagation data and prediction methods required for the design of terrestrial line-of-sight systems," ITU-R, 2015.

[18] S. Sesia, M. Baker, and I. Toufik, LTE-the UMTS long term evolution: from theory to practice. John Wiley \& Sons, 2011.

[19] G. Das, R. Fraser, A. Lóopez-Ortiz, and B. Nickerson, "On the discrete unit disk cover problem," International Journal of Computational Geometry \& Applications, vol. 22, no. 05, pp. 407-419, 2012.

[20] H. W. Kuhn and A. W. Tucker, "Nonlinear programming," in Traces and emergence of nonlinear programming. Springer, 2014, pp. 247-258.

[21] E. Kalantari, M. Z. Shakir, H. Yanikomeroglu, and A. Yongacoglu, "Backhaul-aware robust 3D drone placement in 5G+ wireless networks," in ICC Workshops. IEEE, 2017, pp. 109-114. 